\title{
UV-Initiated Polymerization of Cationic Polyacrylamide: Synthesis, Characterization, and Sludge Dewatering Performance
}

\author{
Huaili Zheng, ${ }^{1}$ Yongjun Sun, ${ }^{1}$ Xiaomin Tang, ${ }^{1}$ Mingzhuo Tan, ${ }^{2}$ Jiangya Ma, \\ Wei Chen, ${ }^{1}$ and Yong Liao ${ }^{1}$ \\ ${ }^{1}$ Key laboratory of the Three Gorges Reservoir Region's Eco-Environment, State Ministry of Education, Chongqing University, \\ Chongqing 400045, China \\ ${ }^{2}$ GuangDong Wealth Environmental Protection Co., Ltd., Guangdong 529000, China
}

Correspondence should be addressed to Yongjun Sun; sunyongjun008@163.com

Received 6 August 2013; Accepted 3 October 2013

Academic Editors: K. F. Chang, X. Luo, and R. A. Smith

Copyright (c) 2013 Huaili Zheng et al. This is an open access article distributed under the Creative Commons Attribution License, which permits unrestricted use, distribution, and reproduction in any medium, provided the original work is properly cited.

$\mathrm{P}(\mathrm{AM}-\mathrm{DAC}-\mathrm{BA})$ was synthesized through copolymerization of acrylamide (AM), acryloyloxyethyl trimethyl ammonium chloride (DAC), and butylacrylate (BA) under ultraviolet (UV) initiation using response surface methodology (RSM). The influences of light intensity, illumination time, and photoinitiator concentration on the intrinsic viscosity $[\eta]$ of $\mathrm{P}(\mathrm{AM}-\mathrm{DAC}-\mathrm{BA})$ were investigated. RSM model based on the influencing data was established for optimizing synthetic conditions. It was found that, at light intensity $1491.67 \mu \mathrm{w} \cdot \mathrm{cm}^{-2}$, illumination time $117.89 \mathrm{~min}$, and photoinitiator concentration $0.60 \%$, there was a better material performance achieved. Thus $\mathrm{P}(\mathrm{AM}-\mathrm{DAC}-\mathrm{BA})$ prepared under the above conditions showed excellent dewatering performance that, with $40 \mathrm{mg} \cdot \mathrm{L}^{-1} \mathrm{P}(\mathrm{AM}-\mathrm{DAC}-\mathrm{BA})$ at $\mathrm{pH}$, the residual turbidity of supernatant and the dry solid content were up to $38 \mathrm{NTU}, 28.5 \%$, respectively.

\section{Introduction}

In recent years, the mount of sewage treated by municipal wastewater treatment plant increases rapidly, resulting in a large amount of excess activated sludge generated, whose moisture content is up to $99.5 \%$, and the sludge containing organic matter and noxious substances will harm the environment and must be handled properly [1]. Because waste activated sludge is a colloidal system in which small sludge particles form a stable suspension in water and are very difficult to be separated from the water phase, the activated sludge must be pretreated before its mechanical dewatering. To improve the sludge dewatering, flocculants are usually added before dewatering, which contact with sludge particles through flocculation process to change the surface charge of sludge particles, reducing electrostatic repulsion between sludge particles to flocculate and aggregate into large flocs. Floccultaion-coagulation process is a very effective way to improve its dewatering performance and achieve the purposes of sludge dewatering.

Currently sludge conditioners used by municipal wastewater treatment plant are cationic polyacrylamides (CPAMs) [2]. CPAMs have many advantages including low dosage, small amount of sludge produced after flocculation, and being less susceptible to both salt and $\mathrm{pH}$. Flocculationcoagulation process could increase the floc settling velocity flocculated by CPAM and make sludge easy to dewater [3]. CPAMs are usually initiated by heat, microwave, rays, and ultraviolet (UV) [4]. UV-initiated polymerization is efficient, energy-saving, and friendly to environment.

Response surface methodology (RSM) is used to optimize the testing program or the relationship between indicators and factors in mathematical model, using multiple regression equation to fit the second factor and the functional relationship between indicators, through the regression equation analysis to find the optimal process parameters $[5,6]$. 
Box-Behnken design was used to optimize the synthesis conditons $[7,8]$. The appliation of Box-Behnken method in wastewater treatment had received a lot of attention, but its application in optimizing the synthesis process of flocculants was rarely studied.

In order to enhance the dewaterability of the wasteactivated sludge, $\mathrm{P}(\mathrm{AM}-\mathrm{DAC}-\mathrm{BA})$ in this study was synthesized through UV-initiated polymerization with AM, DAC, and BA as monomers.

To obtain an optimum synthetic condition, the effect of photoinitiator concentration, incident light intensity, and illumination time on intrinsic viscosity was investigated, and using these investigated data, the response surface methodology (RSM) was fitted to analyze their mutual effect and attain a better condition for preparation of $\mathrm{P}$ (AM-DAC-BA). The $\mathrm{P}(\mathrm{AM}-\mathrm{DAC}-\mathrm{BA})$ used in sludge dewatering showed that the dewaterability of waste activated sludge was affected by flocculant dosage and $\mathrm{pH}$ significantly.

\section{Experimental}

2.1. Experimental Material and Instruments. The acrylamide (AM) (Chongqing Lanjie Tap Water Company, Chongqing, China), butyl acrylate (BA) (Tianjin Guangfu Fine Chemical Research Institute, Tianjin, China), acryloyloxyethyltrimethyl ammonium chloride (DAC, 80\% in water) (Guangchuangjing IMP. EXP. Co., Ltd., Shanghai, China), and the photoinitiator 2,2'-azobis(2-methylpropionamide) dihydrochloride (V-50) (Ruihong biological technology, Shanghai, China) were purchased. All the reagents used in this experiment were without further purification. Commercially available cationic polyacrylamide (CPAM) was sourced from Shandong Polymeibio-Chemicals Co., Ltd., China.

Some instruments, such as UV-A ultraviolet ray intensity meter (Beijing Normal University Photoelectric Instrument Factory, China), TU-1901 double beam ultraviolet and visible spectrophotometer (Beijing Purkinje General Instrument Co., Ltd., China), 550Series II infrared spectrometer (BRUKER 4 Company, Switzerland), were used in this study.

2.2. Synthesis of $P(A M-D A C-B A)$. A predetermined mass ratio of $\mathrm{AM}, \mathrm{DAC}$, and $\mathrm{BA}$ was added into the quartz jar, then adding a certain amount of deionized water, surfactant, and urea. The reagent was mixed and stirred, and then the reaction solution was bubbled with nitrogen gas for $30 \mathrm{~min}$ at room temperature, and, subsequently, the predetermined photoinitiator dosage was added. The reaction vessel was exposed to radiation for $120 \mathrm{~min}$ with a $500 \mathrm{~W}$ high pressure mercury lamp at ambient temperature. After radiation, the product was purified with acetone and ethanol and then was dried and made into powder. Based on the results of the optimized design by response surface method (RSM), the ranges of synthesis condition were listed in Table 2.

2.3. Determination of Intrinsic Viscosity [ $\eta]$. The intrinsic viscosity of CPAM was determined by one point method; the detailed method and operating steps were shown in "the determination method of intrinsic viscosity of polyacrylamide" (GB-T12005.1-1989).

2.4. Sludge Dewatering Experiments. Experimental sludge was collected from sludge storage tank of Chongqing Baihan wastewater treatment plant; the water content of which was $98.56 \%, \mathrm{pH}$ value was 7.56-7.62, and the sludge with fine particles was brown and odor. The density of sludge was $1.02 \mathrm{~g} / \mathrm{mL}$.

In sludge dewatering experiments, the $\mathrm{P}(\mathrm{AM}-\mathrm{DAC}-\mathrm{BA})$ was added into a $250 \mathrm{~mL}$ beaker with $200 \mathrm{~mL}$ sludge included. The $\mathrm{pH}$ of the sludge was adjusted with $0.1 \mathrm{~mol} / \mathrm{L} \mathrm{NaOH}$ and $0.1 \mathrm{~mol} / \mathrm{L} \mathrm{HCl}$. Subsequently, it was rapidly mixed by stirring at $120 \mathrm{rpm}$ for $30 \mathrm{~s}$, followed by a $10 \mathrm{~min}$ quiescent settling time. Then, the supernatant was extracted from the beaker $1 \mathrm{~cm}$ below the solution surface by a syringe for turbidity measurement. Sludge samples were filtered through a vacuum suction filter machine at the pressure of $0.05 \mathrm{MPa}$. The dry solid content (DS) was evaluated by the following equation:

$$
\mathrm{DS}=\frac{W_{1}}{W_{2}} \times 100 \%,
$$

where $W_{1}(\mathrm{~g})$ is the weight of filter cake at the end of filtration and $W_{2}(\mathrm{~g})$ is the weight of filter cake after drying at $105^{\circ} \mathrm{C}$ for $24 \mathrm{~h}$.

\section{Results and Discussion}

3.1. Experiments Design and Results of Regression Model Equation. In order to optimize synthetic condition, establish the model for UV-induced polymerization of P(AM-DAC$\mathrm{BA})$ and investigate the UV effect on $[\eta]$ of $\mathrm{P}(\mathrm{AM}-\mathrm{DAC}-$ $\mathrm{BA})$; three significant levels of ultraviolet factors and $[\eta]$, which had a strong impact on dewaterability efficiency of waste sludge, were selected for the statistical method, where Box-Behnken experimental design was used to investigate the mutual influence among the impact factors on intrinsic viscosity of P(AM-DAC-BA) $[9,10]$. The factor levels selected, as shown in Table 1, indicated that the upper level corresponds to +1 , the lower level to -1 , and the basic level to zero. The experimental result was analyzed using professional software, Design-Expert V8.0.6.

On the basis of response surface methodology, BoxBehnken experimental designs were selected for optimizing and modeling of the preparation of $\mathrm{P}(\mathrm{AM}-\mathrm{DAC}-\mathrm{BA})$, with $X_{1}, X_{2}$, and $X_{3}$ representing photoinitiator concentration, incident light intensity, and illumination time, respectively. Experimental ranges and levels of the independent test variables were shown in Table 2. Seventeen observed responses were used to calculate the model using Box-Behnken method. From the experimental data, the quadratic regression models were established using Design Expert V8.0.6, as shown in

$$
\begin{aligned}
{[\eta]=} & 1.86+0.11 X_{1}+0.38 X_{2}+0.34 X_{3} \\
& +0.075 X_{1} X_{2}+0.14 X_{1} X_{3} \\
& -0.068 X_{2} X_{3}-0.27 X_{1}^{2}-0.15 X_{2}^{2}-0.32 X_{3}^{2} .
\end{aligned}
$$


TABLE 1: Experimental ranges and levels of the independent test variables.

\begin{tabular}{lccc}
\hline Variables & \multicolumn{3}{c}{ Ranges and levels } \\
& -1 & 0 & +1 \\
\hline $\begin{array}{l}\text { Incident light intensity } \\
\left(\mu \mathrm{w} \cdot \mathrm{cm}^{-2}\right)\left(X_{1}\right)\end{array}$ & 700 & 1200 & 1700 \\
$\begin{array}{l}\text { Illumination time (min) } \\
\left(X_{2}\right)\end{array}$ & 40 & 80 & 120 \\
$\begin{array}{l}\text { Photoinitiator } \\
\text { concentration (wt.\%o })\left(X_{3}\right)\end{array}$ & 0.25 & 0.50 & 0.75 \\
\hline
\end{tabular}

TABLE 2: 3-Factor Box-Behnken design and the value of response function $([\eta])$.

\begin{tabular}{lccccc}
\hline Run & \multicolumn{3}{c}{ Coded variables } & \multicolumn{2}{c}{ Intrinsic viscosity $[\eta]\left(\mathrm{dL} \cdot \mathrm{g}^{-1}\right)$} \\
order & $X_{1}$ & $X_{2}$ & $X_{3}$ & Actual value & Predicted value \\
\hline 1 & 0 & 0 & 0 & 1.85 & 1.86 \\
2 & +1 & 0 & 1 & 1.93 & 1.86 \\
3 & -1 & 0 & 1 & 1.45 & 1.35 \\
4 & 0 & -1 & 1 & 1.36 & 1.42 \\
5 & +1 & +1 & 0 & 2.03 & 2.00 \\
6 & -1 & +1 & 0 & 1.64 & 1.63 \\
7 & +1 & 0 & -1 & 0.81 & 0.90 \\
8 & 0 & 0 & 0 & 1.85 & 1.86 \\
9 & 0 & -1 & -1 & 0.71 & 0.61 \\
10 & -1 & 0 & -1 & 0.90 & 0.96 \\
11 & 0 & +1 & 1 & 1.95 & 2.04 \\
12 & -1 & -1 & 0 & 1.00 & 1.02 \\
13 & 0 & 0 & 0 & 1.80 & 1.86 \\
14 & 0 & +1 & -1 & 1.57 & 1.86 \\
15 & 0 & 0 & 0 & 1.85 & 1.86 \\
16 & +1 & -1 & 0 & 1.09 & 1.09 \\
17 & 0 & 0 & 0 & 1.95 & 1.86 \\
\hline
\end{tabular}

The adequacy of the models was investigated using the analysis of variance (ANOVA) and the results were shown in Table 3. $F$-value of the model was 37.33 and the $P$ value was less than 0.0001, indicating that the model was significant. In addition, the value of the determination coefficient, $R^{2}=$ 0.9796 , indicated that the sample variation was $97.96 \%$ for $[\eta]$, which was attributed to the independent variables, and only $2.04 \%$ of the total variations were not explained by the model, suggesting a strong correlation between the predicted and the observed values. Furthermore, the analytical result indicated a better precision and reliability of the experiments being carried out. From the above investigation, it showed that the model was adequate for the prediction of intrinsic viscosity.

3.2. Mutual Effect of Parameters. In order to investigate the various factors and their mutual effect on the intrinsic viscosity of $\mathrm{P}(\mathrm{AM}-\mathrm{DAC}-\mathrm{BA})$, the response surface plots were used to display the response as a function of two factors by keeping the third factor constant, and two-dimensional response surface contours were plotted to investigate the mutual effect of operational variables. The response variables were shown in Figure 1.

Figures 1(a) and 1(b) show the intrinsic viscosity as a function of incident light intensity and illumination time, Figures 1(c) and 1(d) show the intrinsic viscosity as a function of incident light intensity and photoinitiator concentration, and Figures $1(\mathrm{e})$ and $1(\mathrm{f})$ show the intrinsic viscosity as a function of illumination time and photoinitiator concentration. Figures 1(a)-1(f) demonstrate the mutual effect of photoinitiator concentration, incident light intensity, and illumination time on the intrinsic viscosity. It indicated that the increase in photoinitiator concentration and illumination time might lead to the increase of the intrinsic viscosity, especially under the condition of higher photoinitiator concentration and longer illumination time. There was a maximum value in the intrinsic viscosity as the incident light intensity increased within the investigated range of incident light intensity.

3.3. Model Validation. In order to obtain the maximum value of the intrinsic viscosity, the response optimizer in the Design Expert V8.0.6 software was used to search for an optimal synthetic condition. The best condition for the intrinsic viscosity of $\mathrm{P}(\mathrm{AM}-\mathrm{DAC}-\mathrm{BA})$ in theory was that photoinitiator concentration was $0.60 \%$, incident light intensity $1491.67 \mu \mathrm{w} / \mathrm{cm}^{2}$, and illumination time $117.89 \mathrm{~min}$, respectively. The predicted results were calculated by the Design Expert V8.0.6 software based on (2). In order to verify the prediction results, the parallel experiments were carried out. The chosen conditions for cationic degree, photoinitiator concentration, incident light intensity, and illumination time are all listed in Table 4, along with the predicted and measured results. As shown in Table 4, the measured intrinsic viscosity was close to the predicted values using the regression model, indicating the suitability of the fitted polynomial models with respect to the experimental data. But this model was just a theoretical model, it could give a good prediction including optimal conditions and general condition.

3.4. FTIR Spectrum. Figure 2 shows the FTIR spectrum of $\mathrm{P}$ (AM-DMDAAC-BA). The absorption bands observed at $3428.07 \mathrm{~cm}^{-1}$ and $1167.45 \mathrm{~cm}^{-1}$ were originated from strong stretching vibration of amino group and carbonyl group of amide in the AM chain, respectively. The bending vibration absorption peaks of $-\mathrm{CH}_{2}-\mathrm{N}^{+}\left(\mathrm{CH}_{3}\right)_{3}$ group of DAC appeared at $1450.72 \mathrm{~cm}^{-1}$. The stretching vibration absorption peak at $1671.85 \mathrm{~cm}^{-1}$ was attributed to $-\mathrm{C}=\mathrm{O}$ group in the $\mathrm{AM}$ and BA [11]. The analytical results of the FTIR spectrum conformed the formation of the terpolymer of AM, DAC, and BA.

\section{Sludge Dewatering Tests}

4.1. Effect of Dosage on Sludge Dewatering Performance. The effect of dosage on the sludge dewatering performance is shown in Figure 3. The residual turbidity decreased with the increase of the flocculant dosage, which reached a minimum at flocculant dosage of $80 \mathrm{mg} \cdot \mathrm{L}^{-1}$. When the dosage was 


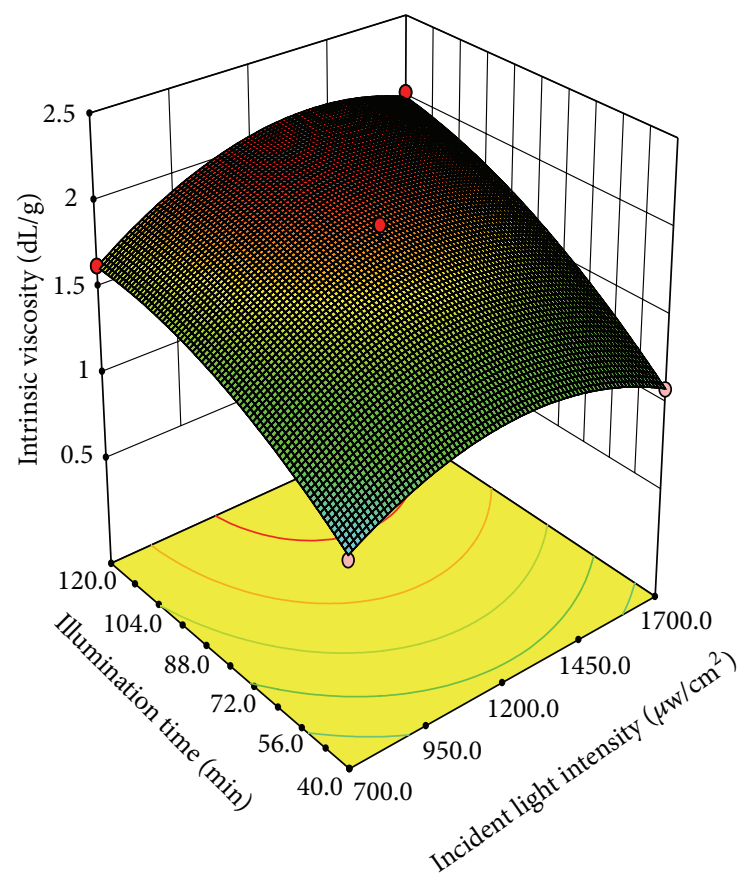

$X_{1}=A$ : incident light intensity

$X_{2}=B$ : illumination time

Actual factor

$C:$ photoinitiator concentration $=0.50$

(a)
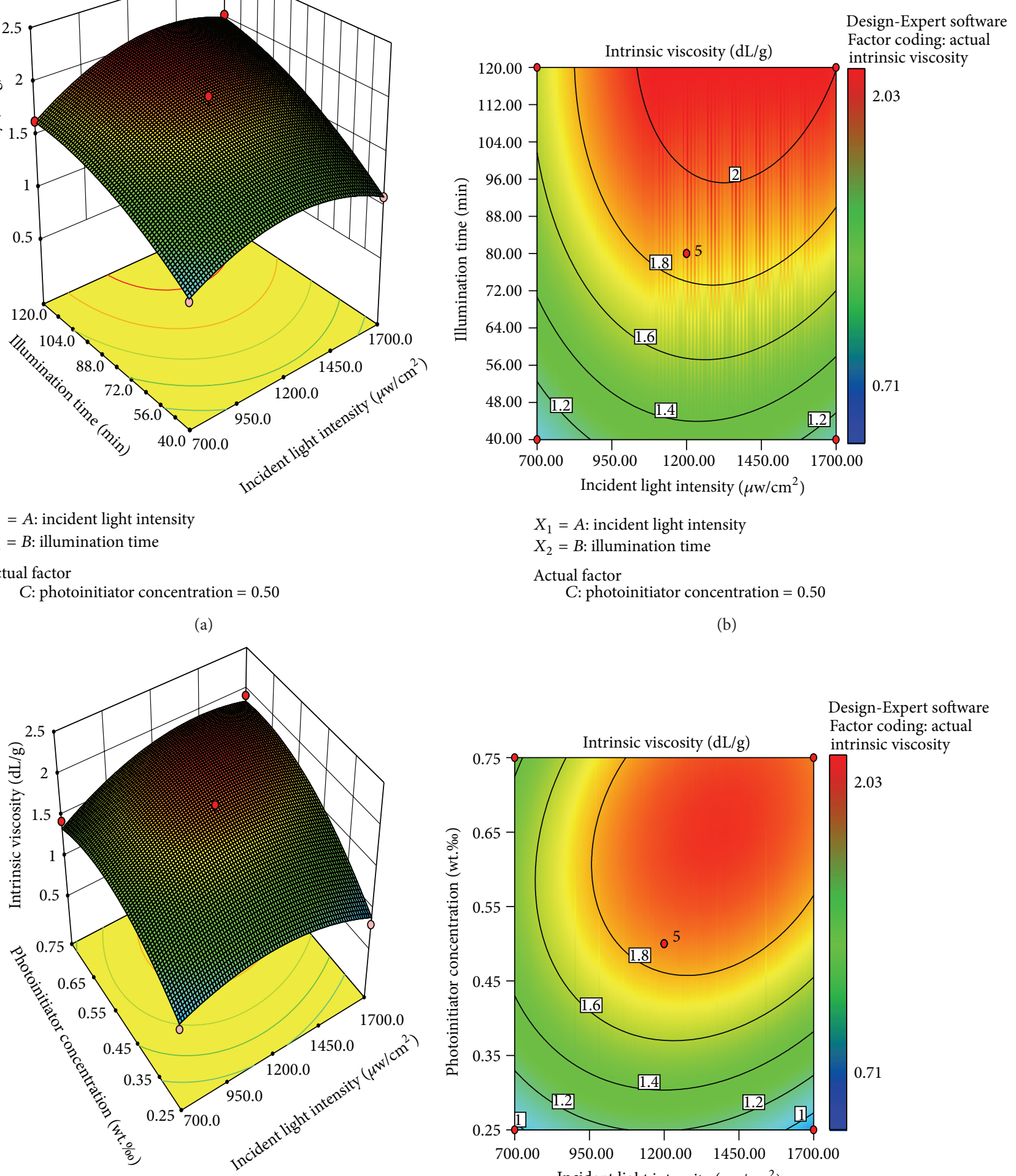

Design points above predicted value

Design points below predicted value

$X_{1}=A$ : incident light intensity

$X_{2}=$ C: photoinitiator concentration

Actual factor

$B:$ illumination time $=80.00$

$X_{1}=A$ : incident light intensity

$X_{2}=B$ : illumination time

Actual factor

$C:$ photoinitiator concentration $=0.50$

(b)

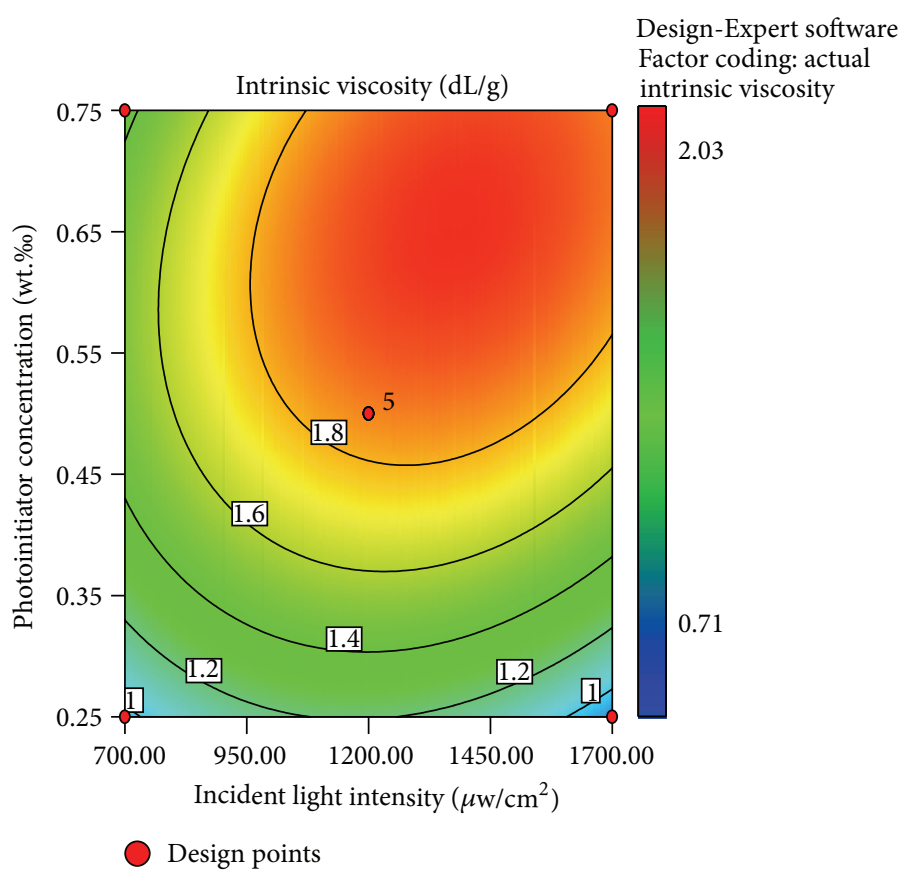

$X_{1}=A$ : incident light intensity

$X_{2}=C$ : photoinitiator concentration

Actual factor

$B$ : illumination time $=80.00$

(c)

FIgure 1: Continued. 


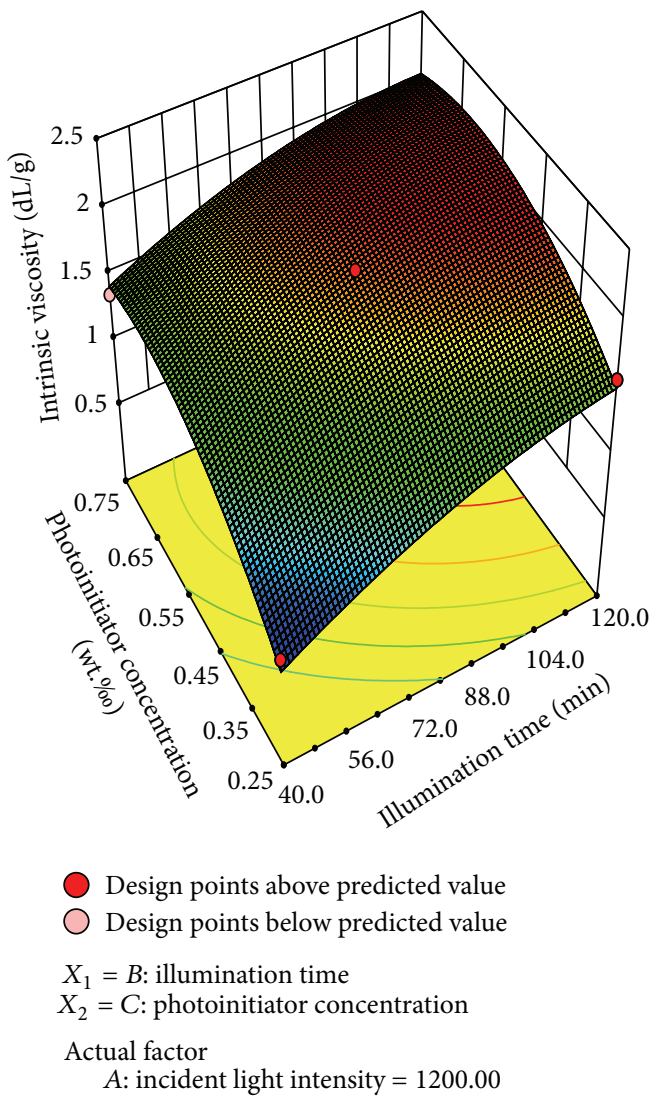

(e)

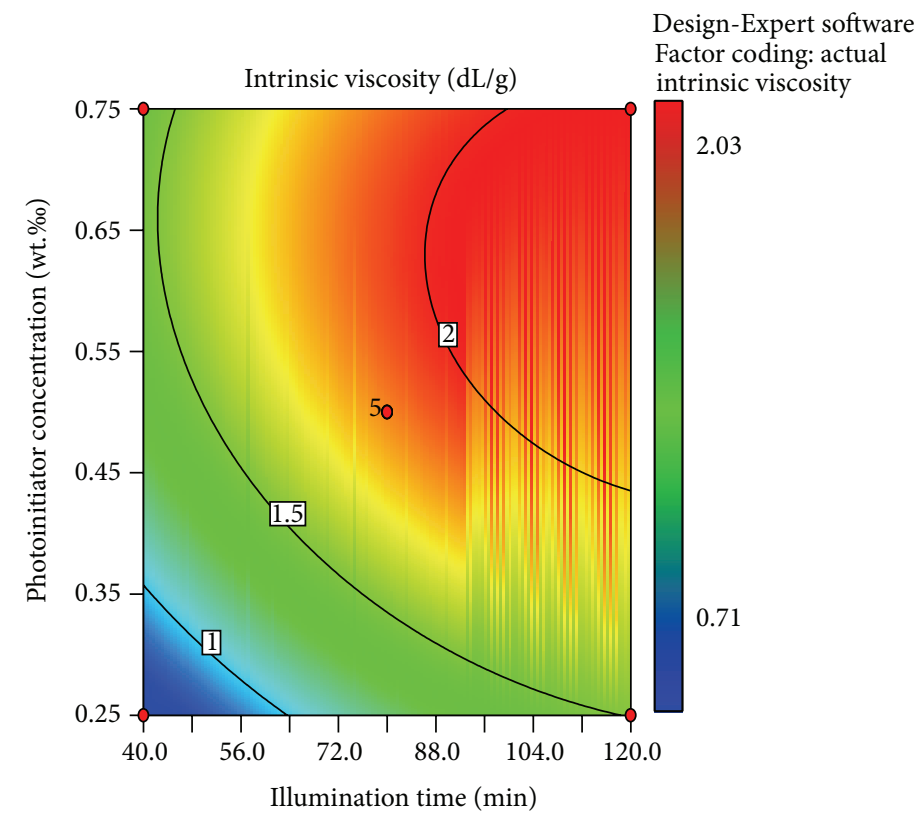

Design points

$X_{1}=B$ : illumination time

$X_{2}=C$ : photoinitiator concentration

Actual factor

$A$ : incident light intensity $=1200.00$

FIGURE 1: Mutual effect of photoinitiator concentration, incident light intensity, and illumination time on intrinsic viscosity $[\eta]$.

TABLE 3: ANOVA results for response parameters.

\begin{tabular}{|c|c|c|c|c|c|}
\hline Source & Sum of squares & Degree of freedom & Mean square & $F$-value & $P$ value \\
\hline Regression & 3.18 & 9 & 0.35 & 37.33 & $<0.0001$ \\
\hline$X_{1}$ & 0.095 & 1 & 0.095 & 9.99 & 0.0159 \\
\hline$X_{2}$ & 1.15 & 1 & 1.15 & 121.12 & $<0.0001$ \\
\hline$X_{3}$ & 0.91 & 1 & 0.91 & 96.17 & $<0.0001$ \\
\hline$X_{1} X_{2}$ & 0.022 & 1 & 0.022 & 2.37 & 0.1672 \\
\hline$X_{1} X_{3}$ & 0.081 & 1 & 0.081 & 8.57 & 0.0221 \\
\hline$X_{2} X_{3}$ & 0.018 & 1 & 0.018 & 1.92 & 0.2080 \\
\hline$X_{1}^{2}$ & 0.31 & 1 & 0.31 & 33.00 & 0.0007 \\
\hline$X_{2}^{2}$ & 0.092 & 1 & 0.092 & 9.67 & 0.0171 \\
\hline$X_{3}^{3}$ & 0.42 & 1 & 0.42 & 44.09 & 0.0003 \\
\hline Residual & 0.066 & 7 & 0.009475 & & \\
\hline Lack of fit & 0.054 & 3 & 0.018 & 6.04 & 0.0575 \\
\hline Pure error & 0.012 & 4 & 0.003 & & \\
\hline Total & 3.25 & 16 & & & \\
\hline$R^{2}$ & 0.9796 & & & & \\
\hline$R_{\text {adj }}^{2}$ & 0.9534 & & & & \\
\hline
\end{tabular}


TABLE 4: Measured and calculated values for confirmation experiments.

\begin{tabular}{lcccc}
\hline Run & $\begin{array}{c}\text { Photoinitiator } \\
\text { concentration }(w t . \%)\end{array}$ & $\begin{array}{c}\text { Incident light } \\
\text { intensity }\left(\mu \mathrm{w} \cdot \mathrm{cm}^{-2}\right)\end{array}$ & $\begin{array}{c}\text { Illumination time }(\mathrm{min}) \\
\eta]\left(\mathrm{dL} \cdot \mathrm{g}^{-1}\right)\end{array}$ & $\begin{array}{c}\text { Measured } \\
\text { Calculated }\end{array}$ \\
\hline 18 & 0.66 & 1422.2 & 102.2 & $2.15 \pm 0.1$ \\
19 & 0.51 & 1407.5 & 101.7 & $1.99 \pm 0.1$ \\
20 & 0.62 & 1285.8 & 88.3 & $1.89 \pm 0.07$ \\
\hline
\end{tabular}

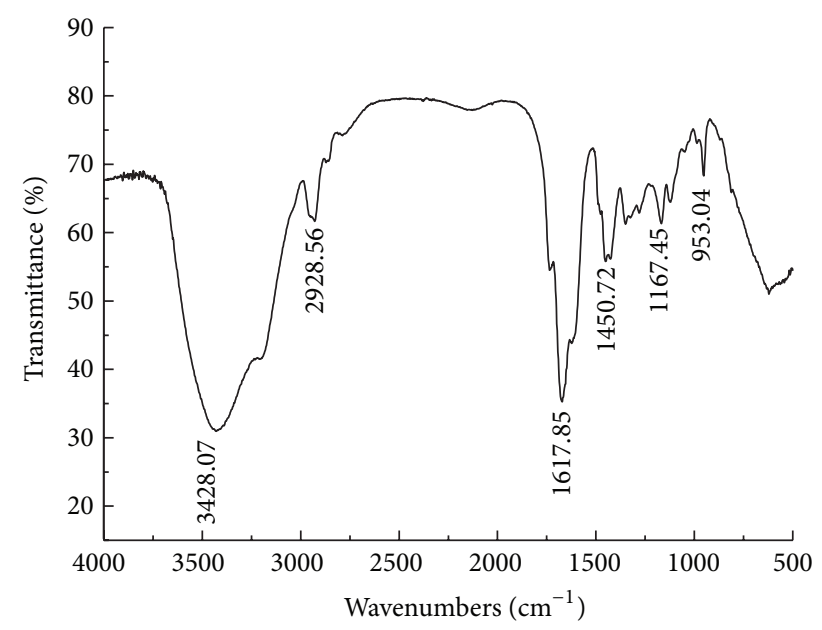

FIGURE 2: FTIR spectrum of P(AM-DMDAAC-BA).

higher than $80 \mathrm{mg} \cdot \mathrm{L}^{-1}$, the residual turbidity increased. With increasing dosage, the dry solid content distinctly decreased firstly and then increased gradually. When the dosage was $40 \mathrm{mg} \cdot \mathrm{L}^{-1}$, the lowest DS of $28.4 \%$ was obtained.

When the dosage was low, the flocculant could not neutralize negatively charged particles, and the sludge flocs were small and friable, not sufficient to improve sludge dewatering performance. So the supernatant turbidity was high and DS was low. When the dosage was too high, the flocculant caused flocculation system positively charged, increasing repulsion between flocs and leading to the restabilization of the flocs $[12,13]$. Excessive dosage would increase the viscosity of the water in sludge, making it not easy to filter. Finally, the dry solid content of sludge cake was reduced [14]. Therefore, an appropriate flocculant dosage of $40 \mathrm{mg} \cdot \mathrm{L}^{-1}$ for dewatering of the waste activated sludge was necessary.

4.2. Effect of $p H$ on Sludge Dewatering Performance. Figure 4 shows the effect of $\mathrm{pH}$ on the sludge dewatering performance. With the increase of the $\mathrm{pH}$, the residual turbidity of the supernatant decreased significantly, but increasing $\mathrm{pH}$ further would cause an increase. In contrary, as increasing the $\mathrm{pH}$ the dry solid content increased and an obvious decrease was found in higher $\mathrm{pH}$ value. The experimental results showed that the $\mathrm{pH}$ between 6 and 8 favored the sludge dewatering where the dry solid content of the filter cake did not change largely.

Under the strong acid and alkaline condition, the sludge dewatering performance was deteriorated resulting in a small

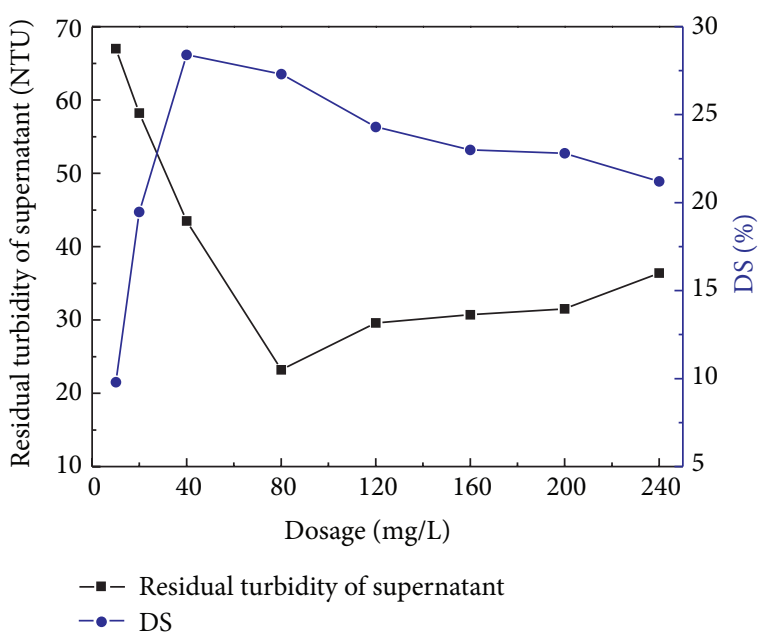

FIGURE 3: Effect of dosage on sludge dewatering performance.

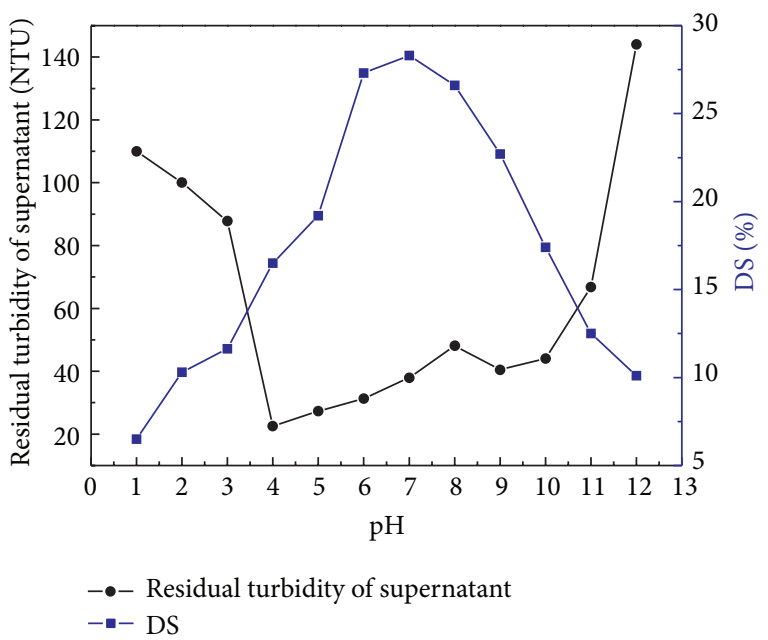

FIGURE 4: Effect of $\mathrm{pH}$ on sludge dewatering performance.

floc and a small volume of supernatant. At low $\mathrm{pH}, \mathrm{H}^{+}$ on the surface of colloidal sludge increased the electrostatic repulsion between sludge particles, leading to the increase of specific resistance of sludge [15]. When the $\mathrm{pH}$ was too high, sludge particle surface would be negatively charged. The drawback was for the flocculant studied that neutralizing the negative charge of the surface of the sludge particles completely was difficult since the repulsion increased between particles would result in an increase in the specific resistance of sludge $[16,17]$. As shown in Figure 4, it indicated that 
TABLE 5: Comparison of dewatering performance between P(AMDAC-BA) and CPAM.

\begin{tabular}{lcccc}
\hline & \multicolumn{3}{c}{ DS (\%) } & \multicolumn{3}{c}{$\mathrm{pH}$} \\
Flocculant & Dosage $(\mathrm{mg} / \mathrm{L})$ & 4 & 7 & 11 \\
\hline P(AM-DAC-BA) & \multirow{2}{*}{40} & 16.5 & 28.4 & 12.5 \\
CPAM & & 13.2 & 26.5 & 8.1 \\
\hline
\end{tabular}

the sludge dewatering experiment using $\mathrm{P}(\mathrm{AM}-\mathrm{DAC}-\mathrm{BA})$ was conducted well at the $\mathrm{pH}$ of 6-8.

In addition, comparison of dewatering performance between P(AM-DAC-BA) and CPAM was shown in Table 5. As listed in Table 5, DS dewatered by $\mathrm{P}(\mathrm{AM}-\mathrm{DAC}-\mathrm{BA})$ was higher than that dewatered by CPAM. It was evident that the dewatering performance of $\mathrm{P}(\mathrm{AM}-\mathrm{DAC}-\mathrm{BA})$ was better than CPAM on the $\mathrm{pH}$ values considered at dosage of $40 \mathrm{mg} / \mathrm{L}$. Dewatering tests demonstrated the superiority of $\mathrm{P}(\mathrm{AM}-$ DAC-BA) over CPAM.

\section{Conclusions}

Flocculant, $\mathrm{P}(\mathrm{AM}-\mathrm{DAC}-\mathrm{BA})$, was synthesized in this study through UV-initiated polymerization using AM, DAC, and $\mathrm{AM}$ as monomers. The best synthetic conditions that were optimized by fitting RSM model while setting the photoinitiator concentration from 0.025 to $0.075 \mathrm{wt} \%$, incident light intensity from 700.0 to $1700.0 \mu \mathrm{w} \cdot \mathrm{cm}^{2}$, and illumination time from 40 to $120 \mathrm{~min}$ were given that the cationic degree, photoinitiator concentration, light intensity, and illumination time were $21.94 \%, 0.6 \%, 1491.67 \mu \mathrm{w} \cdot \mathrm{cm}^{-2}$, and $117.89 \mathrm{~min}$, respectively. FTIR provided the support for the formation of $\mathrm{P}(\mathrm{AM}-\mathrm{DAC}-\mathrm{BA})$. In the dewatering experiments of the waste sludge, it presented a good sludge dewatering performance that, with $40 \mathrm{mg} \cdot \mathrm{L}^{-1} \mathrm{P}(\mathrm{AM}-\mathrm{DAC}-\mathrm{BA})$ at $\mathrm{pH}$ 7, the residual turbidity of supernatant and dry solid content were up to 38 NTU and 28.5\%, respectively.

\section{Conflict of Interests}

The authors declare that there is no conflict of interests regarding the publication of this paper.

\section{Acknowledgments}

This research was supported by the National Natural Science Foundation of China (Project nos. 21177164 and 51078366).

\section{References}

[1] W. Wang, Y. Luo, and W. Qiao, "Possible solutions for sludge dewatering in China," Frontiers of Environmental Science and Engineering in China, vol. 4, no. 1, pp. 102-107, 2010.

[2] E. Neyens, J. Baeyens, R. Dewil, and B. De Heyder, "Advanced sludge treatment affects extracellular polymeric substances to improve activated sludge dewatering," Journal of Hazardous Materials, vol. 106, no. 2-3, pp. 83-92, 2004.
[3] G. Zhu, H. Zheng, Z. Zhang, T. Tshukudu, P. Zhang, and X. Xiang, "Characterization and coagulation-flocculation behavior of polymeric aluminum ferric sulfate (PAFS)," Chemical Engineering Journal, vol. 178, pp. 50-59, 2011.

[4] J. Zhu, H. Zheng, Z. Jiang et al., "Synthesis and characterization of a dewatering reagent: cationic polyacrylamide (P(AM-DMC-DAC) ) for activated sludge dewatering treatment," Desalination and Water Treatment, vol. 51, no. 13-15, pp. 2791-2801, 2013.

[5] S. Sadri Moghaddam, M. R. Alavi Moghaddam, and M. Arami, "Coagulation/flocculation process for dye removal using sludge from water treatment plant: optimization through response surface methodology," Journal of Hazardous Materials, vol. 175, no. 1-3, pp. 651-657, 2010.

[6] M. A. Bezerra, R. E. Santelli, E. P. Oliveira, L. S. Villar, and L. A. Escaleira, "Response surface methodology (RSM) as a tool for optimization in analytical chemistry," Talanta, vol. 76, no. 5, pp. 965-977, 2008.

[7] G. Zhu, H. Zheng, W. Chen, W. Fan, P. Zhang, and T. Tshukudu, "Preparation of a composite coagulant: polymeric aluminum ferric sulfate (PAFS) for wastewater treatment," Desalination, vol. 285, pp. 315-323, 2012.

[8] T. K. Trinh and L. S. Kang, "Application of response surface method as an experimental design to optimize coagulation tests," Environmental Engineering Research, vol. 15, no. 2, pp. 6370, 2010.

[9] H. Zheng, G. Zhu, S. Jiang et al., "Investigations of coagulationflocculation process by performance optimization, model prediction and fractal structure of flocs," Desalination, vol. 269, no. 1-3, pp. 148-156, 2011.

[10] J. Ma, H. Zheng, M. Tan et al., "Synthesis, characterization, and flocculation performance of anionic polyacrylamide $\mathrm{P}(\mathrm{AM}$ AA-AMPS)," Journal of Applied Polymer Science, vol. 129, no. 4, pp. 1984-1991, 2013.

[11] H. Shang, J. Liu, Y. Zheng, and L. Wang, "Synthesis, characterization, and flocculation properties of poly(acrylamidemethacryloxyethyltrimethyl ammonium chloride- methacryloxypropyltrimethoxy silane)," Journal of Applied Polymer Science, vol. 111, no. 3, pp. 1594-1599, 2009.

[12] H. Zheng, G. Zhu, Q. He et al., "A study on the degradation of direct pink by the low-frequency ultrasonic irradiation," Water Science and Technology, vol. 62, no. 4, pp. 829-836, 2010.

[13] N. Buyukkamaci and E. Kucukselek, "Improvement of dewatering capacity of a petrochemical sludge," Journal of Hazardous Materials, vol. 144, no. 1-2, pp. 323-327, 2007.

[14] H. Zheng, Y. Pan, and X. Xiang, "Oxidation of acidic dye Eosin $\mathrm{Y}$ by the solar photo-Fenton processes," Journal of Hazardous Materials, vol. 141, no. 3, pp. 457-464, 2007.

[15] G. Zhu, H. Zheng, P. Zhang, Z. Jiao, and J. Yin, "Near-infrared spectroscopy as a potential tool with radial basis function for measurement of residual acrylamide in organic polymer," Environmental Technology, vol. 34, no. 1, pp. 91-99, 2013.

[16] S. K. Dentel and D. Dursun, "Shear sensitivity of digested sludge: comparison of methods and application in conditioning and dewatering," Water Research, vol. 43, no. 18, pp. 4617-4625, 2009.

[17] Y. Chen, H. Yang, and G. Gu, "Effect of acid and surfactant treatment on activated sludge dewatering and settling," Water Research, vol. 35, no. 11, pp. 2615-2620, 2001. 

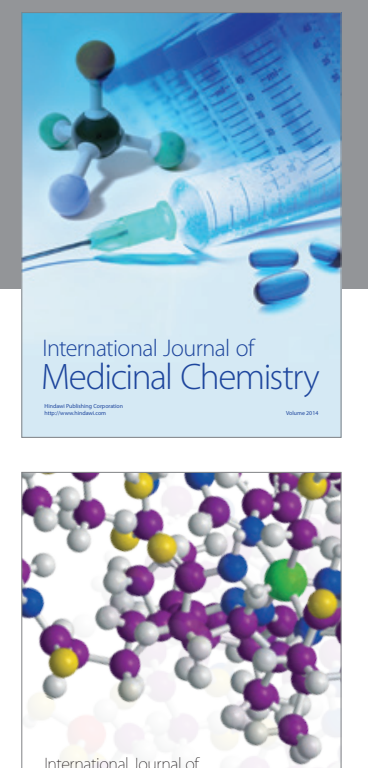

\section{Carbohydrate} Chemistry

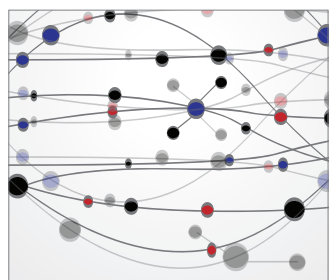

The Scientific World Journal
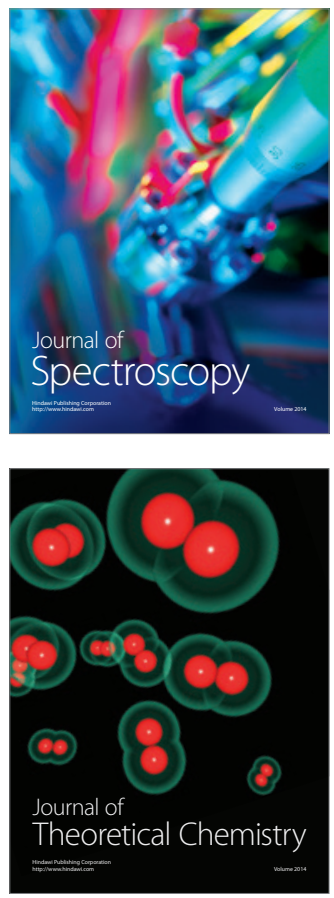
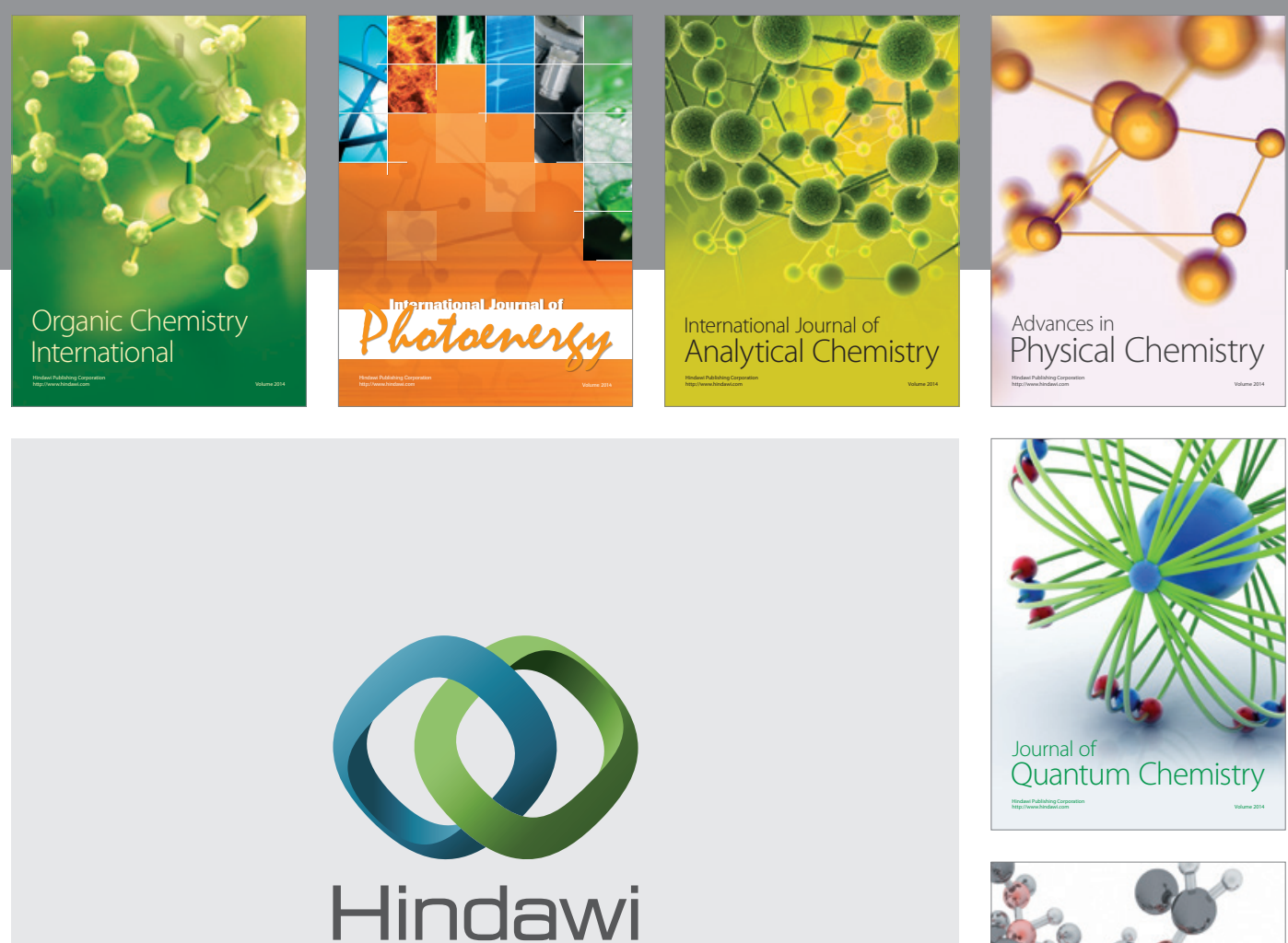

Submit your manuscripts at

http://www.hindawi.com

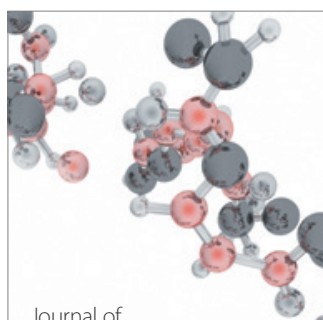

Analytical Methods

in Chemistry

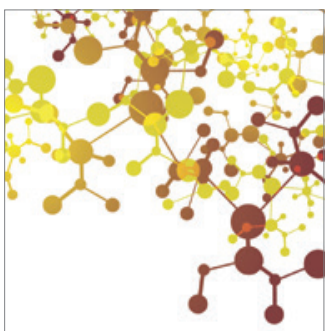

Journal of

Applied Chemistry

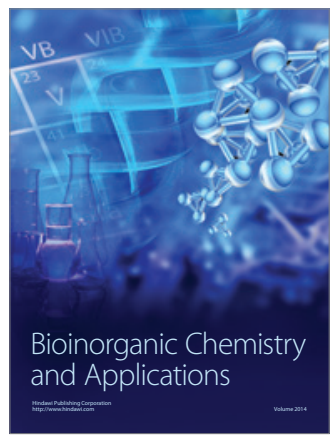

Inorganic Chemistry
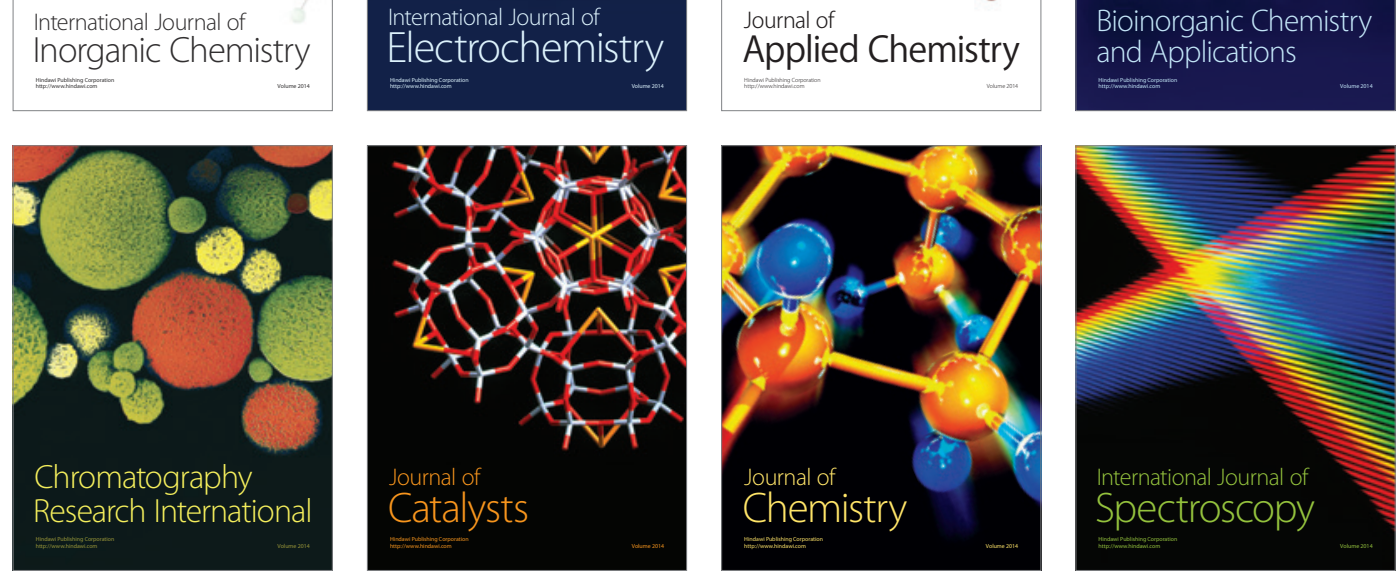